\title{
Synthesis, Properties, and In Vitro Hydrolytic Degradation of $\operatorname{Poly}(\mathrm{d}, \mathrm{l}$-lactide-co-glycolide-co- $\varepsilon$-caprolactone)
}

\author{
Yixiu Liu, ${ }^{1,2}$ Xizhuang Bai, ${ }^{1,3}$ and A. Liang ${ }^{2}$ \\ ${ }^{1}$ Department of Sports Medicine and Joint Surgery, The First Affiliated Hospital of China Medical University, Shenyang 110001, China \\ ${ }^{2}$ Department of Orthopaedics, The Central Hospital Affiliated to Shenyang Medical College, Shenyang 110024, China \\ ${ }^{3}$ Department of Sports Medicine and Joint Surgery, The People's Hospital of Liaoning Province, Shenyang 110015, China
}

Correspondence should be addressed to Xizhuang Bai; baixizhuang123@163.com

Received 12 January 2016; Revised 3 April 2016; Accepted 14 April 2016

Academic Editor: Matthias Schnabelrauch

Copyright (c) 2016 Yixiu Liu et al. This is an open access article distributed under the Creative Commons Attribution License, which permits unrestricted use, distribution, and reproduction in any medium, provided the original work is properly cited.

\begin{abstract}
Random copolymers of poly(d,l-lactide-co-glycolide-co- $\varepsilon$-caprolactone) (PLGC) were synthesized by the ring-opening polymerization of d,l-lactide (DLLA), glycolide (GA), and $\varepsilon$-caprolactone (CL). The effects of CL on the copolymers were evaluated to prepare suitable copolymers with controlled properties. Our results showed that the CL content significantly influenced the thermal and mechanical properties of the copolymers and that the CL content in compositions could be altered to control properties of random copolymers. The in vitro hydrolytic degradation of the resulting implants showed that the degradation rate of PLGC was lower than that of PLGA, which could markedly reduce acidic degradation products. Finally, we demonstrated that higher CL contents in compositions slowed degradation rates.
\end{abstract}

\section{Introduction}

Osteomyelitis is a common inflammatory bone disease caused by pyogenic bacteria. Today, localized drug therapy is a widely accepted strategy for treating osteomyelitis. Nonbiodegradable polymethylmethacrylate (PMMA) bone cement containing antibiotics has been extensively used as a prophylaxis and for the treatment of bone infections since 1973 [1]. However, previous studies revealed several disadvantages with these treatments. These studies showed that the in vitro release of antibiotics from PMMA is incomplete and poorly controlled [2] and that between $90 \%$ and $95 \%$ of the antibiotic remains trapped in the beads [3]. A second surgery is required for removal of the carriers after treatment because of the nonbiodegradability of PMMA. Moreover, the device creates a physical barrier that prevents the new bone from growing into the defect.

To overcome these disadvantages, significant research was devoted to biodegradable polymeric carriers that used several biodegradable materials for osteomyelitis treatment [4-9]. Most research focused on poly(lactide-co-glycolide) (PLGA). The in vitro and in vivo results of the related research showed that the antibiotic-PLGA microspheres were effective for the treatment of chronic osteomyelitis in animal experimental models [10-12]. In addition, Naraharisetti found that about $60 \%$ of gentamicin can be released from PLGA in approximately 5 to 6 days while the remaining drug is completely released in approximately 30 days [13]. These results demonstrated that PLGA could be a promising material for localized therapy of surgically treated bone infections. However, PLGA degrades relatively fast over a few months and poor cell adhesion and growth were observed on unmodified PLGA structures. Several studies demonstrated that the local pH at the site of PLGA implantation decreased as the acidic degradation products were produced, which is detrimental to cells, drugs, or proteins and can lead to inflammatory reactions [14-16]. Meanwhile, the PLGA are in a glassy state during most of the degradation period due to their relatively higher glass transition temperature $\left(T_{g}\right)$ of about $50^{\circ} \mathrm{C}$ that renders the materials stiff and inflexible. Therefore, the properties of PLGA require urgent modification.

Copolymerization is a successful method of modifying polymer physical properties. $\operatorname{Poly}(\varepsilon$-caprolactone) (PCL) is 
a semicrystalline polymer with high permeability, biocompatibility, and good mechanical properties which biodegrades and is absorbed in vivo $[17,18]$. PCL degrades very slowly. It takes 1 2 years to degrade in vivo because of its relatively hydrophobic character and high crystallinity $[19,20]$. The random incorporation of $\varepsilon$-caprolactone monomer units into the backbone of PLGA decreases the $T_{g}$ of PLGA and causes degradation as well as an increased use of PLGA in biomedical fields.

In this study, we found that the synthesis and characterization of biodegradable copolymers of CL with DLLA and GA (PLGC) produced elastic biodegradable polymers with controllable degradation rates. The structure of the resulting copolymers was verified by proton nuclear magnetic resonance $\left({ }^{1} \mathrm{H}-\mathrm{NMR}\right)$, and the molecular weight and molecular weight distribution were tested by gel permeation chromatography (GPC). Copolymer thermal properties were determined by a differential scanning calorimeter (DSC) and a thermogravimetric analyzer (TGA). The cylindrical PLGC implant was prepared with a hot extrusion and incubated in pH 7.4 PBS to investigate its degradation behavior. The effect of CL content on the mechanical and thermal properties and on the in vitro hydrolytic degradation was evaluated as well.

\section{Experimental Materials}

Monomer d,l-lactide (DLLA) and glycolide (GA) (Daigang Biomaterial Co., Ltd., China) were recrystallized from acetic ether and dried at $37^{\circ} \mathrm{C}$ under reduced pressure before polymerization. Also, $\varepsilon$-caprolactone (CL) was purchased from Sigma-Aldrich, freshly distilled over $\mathrm{CaH}_{2}$ under reduced pressure prior to use. Finally, $\mathrm{Sn}(\mathrm{Oct})_{2}(95 \%)$ was purchased from and used in its original state from Sigma-Aldrich. All other solvents and reagents used were of analytical grades and were purified by standard methods.

2.1. Measurements. The composition and structure of the polymers were determined by an ${ }^{1} \mathrm{H}$ NMR analysis spectrometer (Bruker ARX 600, Switzerland) using $\mathrm{CDCl}_{3}$ solutions with tetramethylsilane as an internal reference. The molecular weights and their distributions ( $\mathrm{Mn}, \mathrm{Mw}$, and polydispersity) were determined by GPC (Waters, USA) with a Waters Model 1515 isocratic high-performance liquid chromatography (HPLC) pump, a Waters Model 2414 differential refractive index detector, and three Waters Styragel chromatographic columns (HT2, HT3, and HT4). THF was used as the solvent and eluent for copolymers at a flow rate of $1 \mathrm{~mL} / \mathrm{min}$ at $35^{\circ} \mathrm{C}$. The phase behaviors were determined with a DSC (TA Q2000, USA) under a nitrogen atmosphere. The samples were run at a heating rate of $10^{\circ} \mathrm{C} / \mathrm{min}$ from $100^{\circ} \mathrm{C}$ to $200^{\circ} \mathrm{C}$ to eliminate the thermal history. Next, a second scan was recorded and the glass transition temperatures $\left(T_{g}\right)$ were measured from the second heating cycle. The thermal stability of the polymers under the nitrogen atmosphere was determined by a TGA (TA Q500, USA) and the samples were heated from room temperature to $600^{\circ} \mathrm{C}$ at a heating rate of $10^{\circ} \mathrm{C} / \mathrm{min}$. The mechanical properties of copolymers were measured using a universal testing machine (Instron
5965, USA). Tests were performed on triplicate samples and the results were averaged. The scanning electron microscopy (SEM) micrographs of the specimens before and after degradation were determined with a microscope (FEI inspect F50, USA). The $\mathrm{pH}$ of the degradation products was monitored by inserting a combination microelectrode (InLabMicro ${ }^{\mathrm{TM}}$, Toledo-Mettler) into the medium. Changes in the medium were monitored with a digital $\mathrm{pH}$ meter $\left(\right.$ FiveEasy ${ }^{\mathrm{TM}}$, ToledoMettler).

2.2. Preparation of PLGC. PLGC was synthesized by bulk ring-opening copolymerization in glass ampoules and purged with dry nitrogen. The mixture of DLLA, GA, and CL were added to glass ampoules under a nitrogen atmosphere, and $\mathrm{Sn}(\mathrm{Oct})_{2}$ was added to the solution in anhydrous toluene $\left(2 \times 10^{-4} \mathrm{~mol}\right.$ of $\mathrm{Sn}(\mathrm{Oct})_{2}$ per mol of monomer $)$. The toluene was removed by evacuation. The ampoules were purged three times with dry nitrogen and heat-sealed using a vacuum. The ampoules were placed an oil bath and reacted at $130^{\circ} \mathrm{C}$ for 24 hours while being vigorously shaken to obtain a homogeneous mixture of the monomers and the catalyst. After the copolymerization, the polymers were discharged and then dissolved in $\mathrm{CHCl}_{3}$, precipitated in excess methanol, washed with methanol, and dried under reduced pressure at $37^{\circ} \mathrm{C}$ until they achieved a constant weight for further analysis. For the mechanical properties study and the in vitro degradation study, cylindrical PLGC implants were prepared by hot extrusion using a twin-screw microcompounder (Haake Minilab II, Germany).

2.3. In Vitro Hydrolytic Degradation. Cylindrical specimens with a length of $20 \mathrm{~mm}$ were weighed and conditioned in pH 7.4 PBS solutions, which were refreshed once a week. The degradation experiments were performed in triplicate at $37^{\circ} \mathrm{C}$ with gentle shaking. At regular time intervals, the polymer specimens were taken from the degradation media and washed with distilled water. After blotting with a tissue, the specimens were weighed and vacuum-dried at $37^{\circ} \mathrm{C}$ to a constant weight. The $\mathrm{pH}$ of the media containing degradation products was measured. The mass loss was calculated according to the following equation:

$$
\text { Mass loss }(\%)=\frac{w_{i}-w_{d}}{w_{i}} \times 100 \text {, }
$$

where $w_{i}$ is the initial weight and $w_{d}$ is the dry weight of the samples.

\section{Results and Discussion}

3.1. Synthesis and Characterization. The PLGC was synthesized by a bulk ring-opening polymerization of DLLA, GA, and CL (Scheme 1). $\mathrm{Sn}(\mathrm{Oct})_{2}$ is preferred for biomedical applications because of its low toxicity [21]. The polymerization reaction was carried out under different conditions by changing the monomers ratio and the results of the copolymerization are given in Table 1 .

Under the conditions mentioned in Table 1, all copolymerization reached high yields (>90\%). The molecular weight 


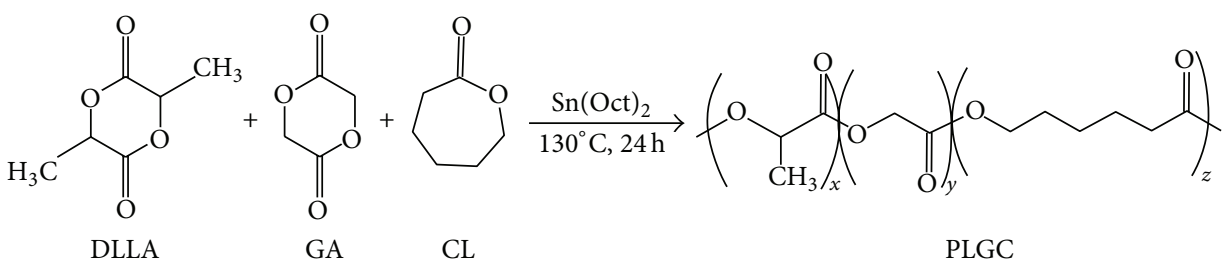

Scheme 1: Synthetic route of PLGC in the presence of $\mathrm{Sn}(\mathrm{Oct})_{2}$.

TABLE 1: Ring-opening copolymerization of DLLA, GA, and CL in the bulk with Sn(Oct) ${ }_{2}$.

\begin{tabular}{|c|c|c|c|c|c|c|c|c|}
\hline \multirow{2}{*}{ Number } & \multicolumn{2}{|c|}{$[\mathrm{DLLA}] /[\mathrm{GA}] /[\mathrm{CL}]$} & \multirow{2}{*}{$\begin{array}{l}\mathrm{Mw}^{\mathrm{b}} \\
\times 10^{4}\end{array}$} & \multirow{2}{*}{$\begin{array}{l}\mathrm{Mn}^{\mathrm{b}} \\
\times 10^{4}\end{array}$} & \multirow{2}{*}{$\mathrm{PDI}^{\mathrm{b}}$} & \multirow{2}{*}{$\begin{array}{c}T_{g}{ }^{\mathrm{d}} \\
{ }^{\circ} \mathrm{C}\end{array}$} & \multirow{2}{*}{$\begin{array}{l}T_{g}{ }^{\mathrm{e}} \\
{ }^{\circ} \mathrm{C}\end{array}$} & \multirow{2}{*}{$\begin{array}{c}T_{d}{ }^{\mathrm{f}} \\
{ }^{\circ} \mathrm{C}\end{array}$} \\
\hline & Feeding & Polymer $^{\mathrm{a}}$ & & & & & & \\
\hline 1 & $50: 50: 0$ & $-^{c}$ & $-^{c}$ & $-^{c}$ & $-^{c}$ & 44.3 & $-^{c}$ & 246.3 \\
\hline 2 & $40: 40: 20$ & $42.07: 38.57: 19.36$ & 18.5 & 14.3 & 1.29 & 21.8 & 20.30 & 268.6 \\
\hline 3 & $30: 30: 40$ & $31.62: 28.18: 40.20$ & 16.1 & 12.4 & 1.30 & -6.2 & -5.17 & 278.7 \\
\hline
\end{tabular}

${ }^{\mathrm{a}}$ Determined by ${ }^{1} \mathrm{H}-\mathrm{NMR}$.

${ }^{\mathrm{b}}$ Calculated from GPC analysis.

${ }^{\mathrm{C}}$ No data for the undissolvable samples.

${ }^{\mathrm{d}}$ Determined from DSC analysis.

${ }^{\mathrm{e}}$ Calculated from FOX equation.

${ }^{\mathrm{f}} T_{d}$ is the $5 \%$ weight loss temperature of thermal degradation.

characterizations were determined by GPC. As displayed in Table 1, all of the prepared copolymers had high molecular weights with narrow molecular weight distributions. As such, it is necessary to accurately regulate the degradation speed rates of the polymers in biomedical applications. Moreover, we demonstrated that the feeding dose significantly influenced the molecular weight of PLGC. As shown in Table 1, the PLGC copolymer containing $0 \mathrm{~mol} \% \mathrm{CL}$ was not dissolved in organic solvents because of its high molecular weight. The molecular weights of the PLGC copolymers with $20 \mathrm{~mol} \%$ and $40 \mathrm{~mol} \% \mathrm{CL}$ content were $1.43 \times 10^{5} \mathrm{~g} / \mathrm{mol}$ and $1.24 \times$ $10^{5} \mathrm{~g} / \mathrm{mol}$. Therefore, molecular weight decreased as the CL content increased.

The structure of the PLGC copolymer was elucidated from the ${ }^{1} \mathrm{H}$ NMR spectra. The ${ }^{1} \mathrm{H}-\mathrm{NMR}$ spectra of PLGA $50 / 50(\mathrm{Mn}=50000), \mathrm{PCL}$, and PLGC with $20 \mathrm{~mol} \% \mathrm{CL}$ content (number 2 in Table 1) are presented in Figure 1. There were three peaks in the PLGA spectrum at $\delta=5.20(\mathrm{H}, \mathrm{Ha})$, $4.77(2 \mathrm{H}, \mathrm{Hc})$, and $1.52(3 \mathrm{H}, \mathrm{Hb})$, respectively [22]. Figure 1 also shows four peaks of proton signals in the PCL spectrum at $\delta=4.06(2 \mathrm{H}, \mathrm{Hd}), 2.31(2 \mathrm{H}, \mathrm{Hg}), 1.60 \sim 1.70(2 \mathrm{H}, \mathrm{He})$, and 1.35 1.43 (2H, Hf) [23]. In the spectrum of PLGC, these signals appeared at $\delta=5.16 \sim 5.26(\mathrm{H}, \mathrm{Ha}), 4.66 \sim 4.89(2 \mathrm{H}, \mathrm{Hc})$, 4.05 4.17 (2H, Hd), 2.30 2.45 (2H, Hg), 1.62 1.66 (4H, He), $1.48 \sim 1.59(3 \mathrm{H}, \mathrm{Hb})$, and $1.26 \sim 1.42(2 \mathrm{H}, \mathrm{Hf})$. As the chemical environment varied in the copolymer, the peak shape was varied, and all the peaks were split into multipeaks [24]. The results of ${ }^{1} \mathrm{H}$ NMR demonstrated that it matched the expected values and indicates the successful synthesis of PLGC.

The $[\mathrm{DLLA}] /[\mathrm{GA}] /[\mathrm{CL}]$ ratios of the copolymer were calculated from the peak areas corresponding to the methine protons of DLLA at $\delta=5.16 \sim 5.26 \mathrm{ppm}$, methylene protons of GA at $\delta=4.66 \sim 4.89 \mathrm{ppm}$, and $\varepsilon$-methylene protons of $\mathrm{CL}$ at $\delta=4.05 \sim 4.17 \mathrm{ppm}$. Under the applied polymerization conditions, the [DLLA]/[GA]/[CL] ratios of the obtained

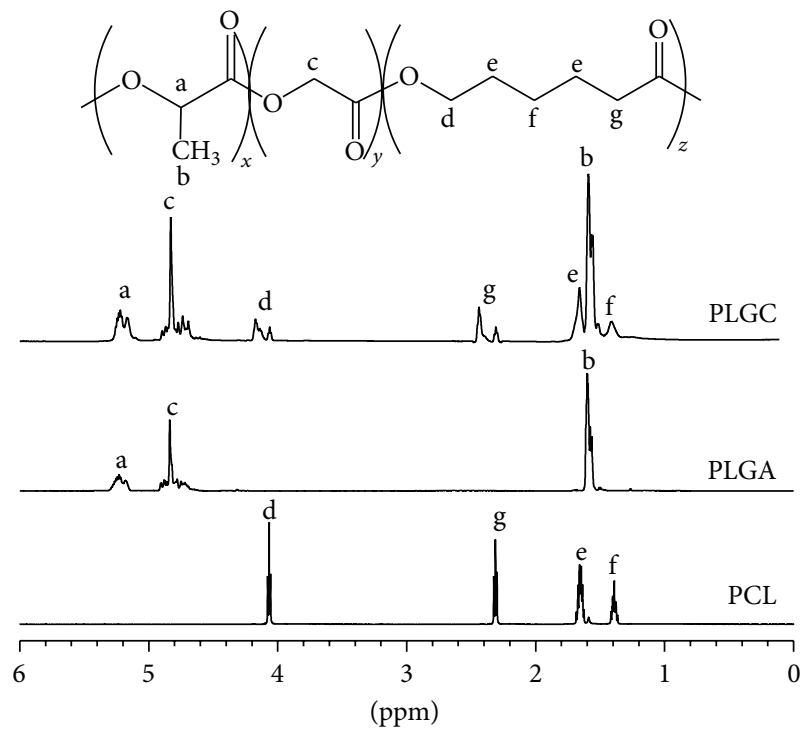

FIGURE 1: The ${ }^{1} \mathrm{H}$ NMR spectra of PLGA 50/50, PCL, and PLGC with 20 mol\% CL content.

copolymers were very close to those in the feeding dose. This indicated a good conversion of monomers and determined that $\mathrm{Sn}(\mathrm{Oct})_{2}$ is effective for the copolymerization of $\mathrm{CL}$ and DLLA.

3.2. Thermal Properties. The glass transition temperature is an important parameter of molecular structure and motion. The phase behaviors of the PLGC copolymers were determined by DSC. The samples were run at a heating rate of $10^{\circ} \mathrm{C} / \mathrm{min}$ from $-100^{\circ} \mathrm{C}$ to $200^{\circ} \mathrm{C}$ to eliminate the thermal history. A second scan was recorded and the glass transition temperature $\left(T_{g}\right)$ was measured from the second heating 


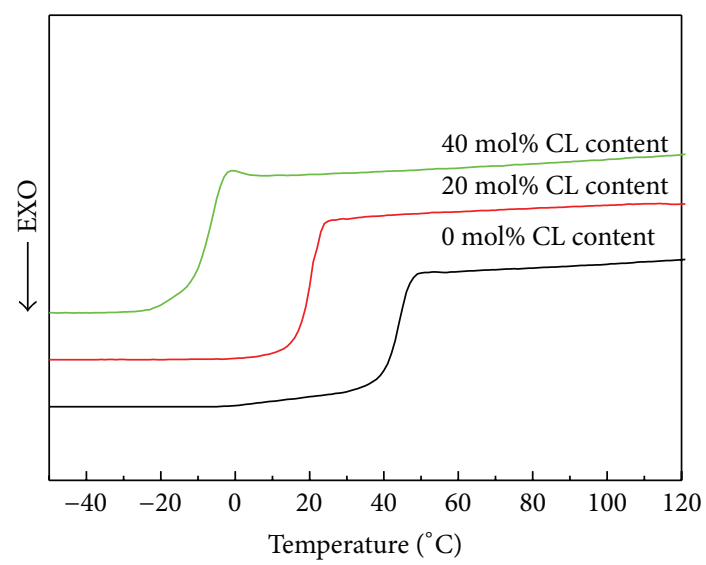

FIgURE 2: The DSC thermograms of PLGC copolymers.

cycle. The data of the thermal properties are also listed in Table 1, and the typical curves are given in Figure 2.

The $T_{g}$ of PLGC was significantly dependent on the copolymers composition. The higher the CL content in composition, the lower the $T_{g}$ of the PLGC copolymers (Table 1). This was attributed to the low $T_{g}$ about $-64^{\circ} \mathrm{C}$ for PCL homopolymers [23, 25]. The copolymer structure can be further verified by DSC analysis. According to the DSC results as shown in Figure 2, all of the copolymers showed a single glass transition temperature, which confirmed the random nature of the PLGC copolymers as the block copolymer has two $T_{g} s$ [26]. As opposed to the homopolymers of PCL and PGA that are semicrystalline, the copolymers were amorphous and the $T_{g}$ values were below body temperature. This allows these flexible elastomeric materials to be rubbery in implant applications for osteomyelitis treatments.

Copolymerization could mean that the copolymers with distinct performances result from the respective homopolymers. For random copolymers, Fox derived the following equation that finds the glass transition temperature of a copolymer based on the glass transition temperatures of the respective homopolymers [27]:

$$
\frac{1}{T_{g}}=\frac{W_{1}}{T_{g_{1}}}+\frac{W_{2}}{T_{g_{2}}}+\frac{W_{3}}{T_{g_{3}}}
$$

where $W_{1}, W_{2}$, and $W_{3}$ are the weight fractions of the DLLA, GA, and CL monomer units in the copolymer, while $T_{g_{1}}, T_{g_{2}}$, and $T_{g_{3}}$ are the $T_{g}(\mathrm{~K}) \mathrm{s}$ of PDLLA, PGA, and PCL homopolymers. For example, the weight fractions for the copolymer ([DLLA]/[GA]/[CL] $=40: 40: 20)$ are $W_{1}=$ $0.4755, W_{2}=0.35212$, and $W_{3}=0.1733$. Substituting these and the $T_{g}$ of $T_{g_{1}}=57^{\circ} \mathrm{C}(330.1 \mathrm{~K})[28], T_{g_{2}}=35^{\circ} \mathrm{C}(308.1 \mathrm{~K})$ [28], and $T_{g_{3}}=-63.8^{\circ} \mathrm{C}(209 \mathrm{~K})$ into Fox equation yields a predicted $T_{g}$ of $20.30^{\circ} \mathrm{C}$. It was interesting to compare the $T_{g}$ values determined by DSC with those calculated from the Fox equation. The experimental results compared to the model matched were as expected, which also indicated that the range of compositions of the copolymers matched the monomers feed ratio.

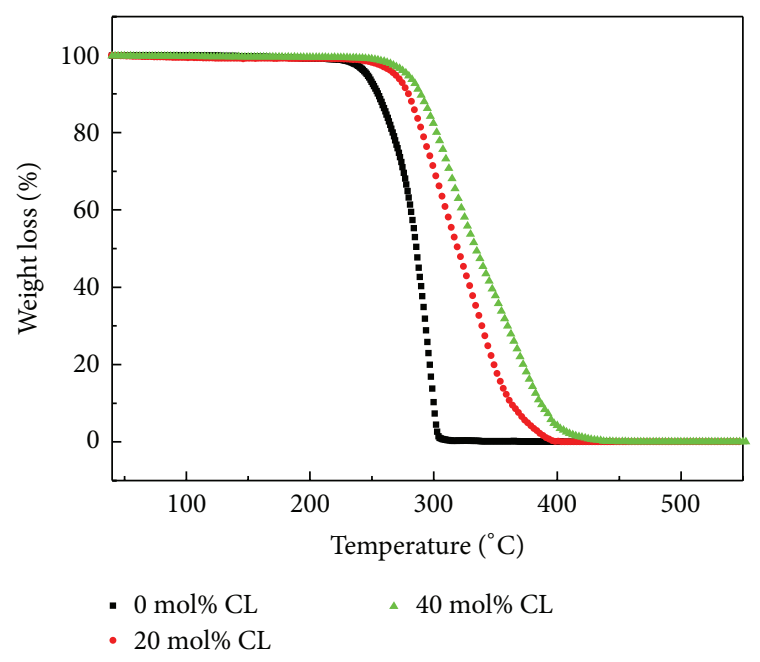

FIgURE 3: The TGA thermograms of PLGC copolymers.

The thermal stability of the resulting PLGC copolymers was characterized in terms of the $5 \%$ weight loss temperature of thermal degradation $\left(T_{d}\right)$. The representative TGA curves of the PLGC copolymers were determined by thermogravimetric analysis in nitrogen (Figure 3). As shown in Table 1, the thermal decomposition temperature of the PLGC copolymers with different compositions was above $200^{\circ} \mathrm{C}$, ranging from $246.3^{\circ} \mathrm{C}$ to $278.8^{\circ} \mathrm{C}$, indicating that all the polymers had good thermal stabilities. The $T_{d}$ of the PLGC increased gradually with increasing CL content in composition, because PCL chains have thermal decomposition temperature higher than $340^{\circ} \mathrm{C}$ [25]. Therefore, the higher the CL content, the higher the thermal stability of the PLGC copolymers.

Our analysis showed that the feeding dose or composition had a great influence on the thermal properties of the copolymers. The synthesized PLGC copolymer with CL content ranging within $20 \sim 40 \mathrm{~mol} \%$ had $T_{g}$ between $-6.2^{\circ} \mathrm{C}$ and $218^{\circ} \mathrm{C}$ with no crystallinity observed. These copolymers became more flexible and elastic at the temperature above those values (such as at $37^{\circ} \mathrm{C}$ of the body temperature) because of their amorphous structures and low glass transition temperatures. The elevated thermal stability with increasing CL content is necessary for producing implants in marrow cavities that are stable with no observation of thermal degradation at the physiological temperature. The stable thermal polymers must be sterilized by traditional techniques such as steam or dry heat, in an efficient and economical manner, without destruction of their structures or changes in their material characteristics.

3.3. Mechanical Properties. We studied the mechanical properties of the cylindrical specimens of the prepared PLGC copolymers. The results are listed in Table 2 with representative stress-strain curves at room temperature shown in Figure 4.

As shown in Table 2, we demonstrated that the CL content in the composition had significant effects on the mechanical properties of the copolymers. The modulus and 
TABLE 2: Mechanical properties of the PLGC copolymers.

\begin{tabular}{|c|c|c|c|c|c|c|}
\hline Number & $\begin{array}{c}\text { CL } \\
\mathrm{mol} \%\end{array}$ & $\begin{array}{c}E^{\mathrm{a}} \\
\mathrm{MPa}\end{array}$ & $\begin{array}{l}\sigma_{m}{ }^{\mathrm{b}} \\
\mathrm{MPa}\end{array}$ & $\begin{array}{c}\varepsilon_{m}{ }^{\mathrm{c}} \\
\%\end{array}$ & $\begin{array}{c}\sigma_{b}^{\mathrm{d}} \\
\mathrm{MPa}\end{array}$ & $\begin{array}{c}\varepsilon_{b}{ }^{\mathrm{e}} \\
\%\end{array}$ \\
\hline 1 & 0 & $3136 \pm 200$ & $51.63 \pm 7.23$ & $3.13 \pm 0.72$ & $24.83 \pm 4.50$ & $37.86 \pm 5.72$ \\
\hline 2 & 20 & $9.19 \pm 1.1$ & $7.41 \pm 0.81$ & $392.5 \pm 52.5$ & $7.17 \pm 1.17$ & $392.5 \pm 52.5$ \\
\hline 3 & 40 & $3.70 \pm 1.16$ & $1.38 \pm 0.09$ & $97.33 \pm 4.62$ & $0.52 \pm 0.17$ & $520 \pm 45.8$ \\
\hline
\end{tabular}

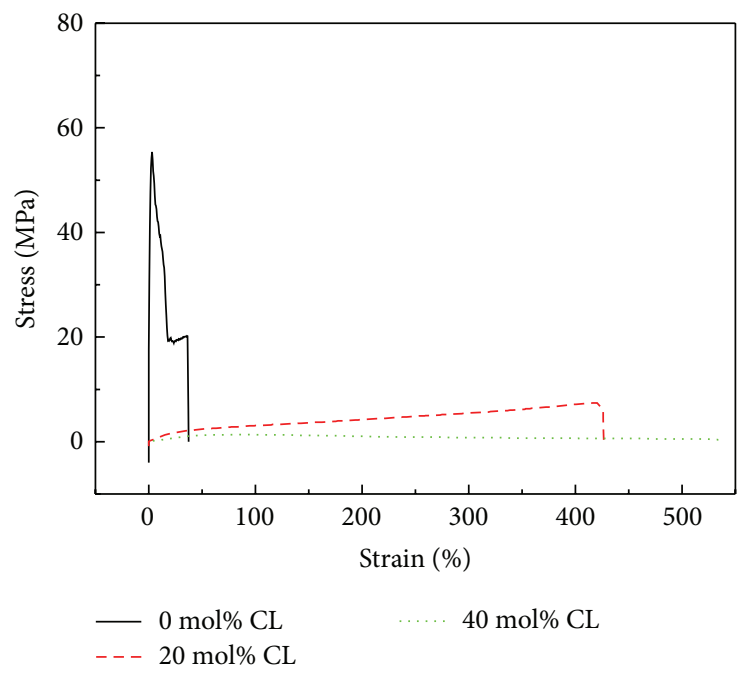

FIgURE 4: The stress-strain curves of the PLGC copolymers.

tensile strength decreased as CL content was introduced in the polymer chain. For example, the modulus and tensile stress decreased from 3136 to $3.7 \mathrm{MPa}$ and from 51.63 to $1.38 \mathrm{MPa}$, while the CL content increased from 0 to $40 \mathrm{~mol} \%$, and the polymers varied from stiff materials to rubber.

3.4. In Vitro Hydrolytic Degradation. To determine the effect of CL content on the degradation behavior of the PLGC, the copolymers containing $20 \mathrm{~mol} \%$ and $40 \mathrm{~mol} \%$ CL were immersed in $\mathrm{pH} 7.4$ phosphate buffer solutions. As a control, the degradation of PLGA $(50 / 50 \mathrm{~mol} \%)$ was similarly buffered. The in vitro hydrolytic degradation of PLGC was then determined as a function of the degradation time and monitored by weight loss, changes in molecular weight, and SEM observation. The changes in copolymer composition during hydrolysis were determined as well via ${ }^{1} \mathrm{H}$ NMR analyses.

Figure 5 showed the weight loss of PLGC copolymers incubated in the PBS buffer medium at $37^{\circ} \mathrm{C}$. In Figure 5, all polymers lost weight gradually and all the copolymers degraded rapidly. The PLGC copolymers containing a CL unit degraded slower than the PLGA (50/50). For example, the PLGC copolymers containing $20 \mathrm{~mol} \%$ and $40 \mathrm{~mol} \%$ CL content lost their masses of $89.08 \%$ and $76.74 \%$ after 7 weeks' in vitro hydrolytic degradation, while the PLGA

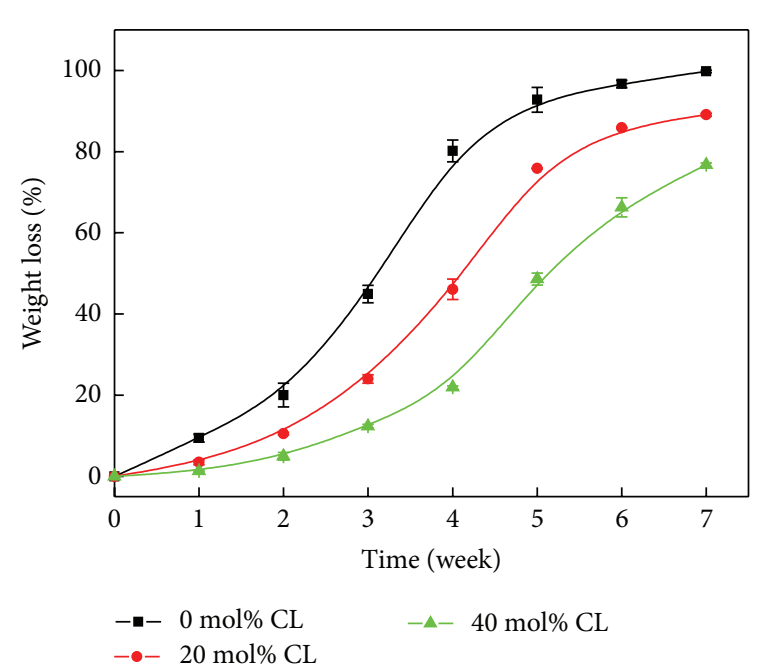

FIGURE 5: The weight loss of PLGC copolymers in vitro as a function of degradation time.

(50/50) degraded in its entirety at the same time point. This was attributed to the fact that the PCL segments contained in the PLGC copolymers are known to degrade very slowly compared with the PLGA chains [29], which results in preferential cleavage along the backbone of the PLGA chains. We concluded that the monomer composition ratio of PLGC affected its hydrolysis and the degradation rate of the PLGC copolymers decreased with elevated CL content in compositions. The results indicated that the introduction of CL content in PLGA chains would delay its degradation, and the degradation rate of PLGC copolymers could be tailored with the CL content to meet the diverse requirements of the different clinical applications.

The composition of the PLGC copolymers changed significantly with degradation (Figure 6). This change was calculated by ${ }^{1} \mathrm{H}$ NMR spectra. The content of [DLLA+GA] in the sample decreased with degradation while the content of CL increased. After 6 weeks of in vitro hydrolytic degradation, the $[\mathrm{DLLA}+\mathrm{GA}]$ content in the PLGC copolymer $(20 \mathrm{~mol} \%$ $\mathrm{CL}$ ) decreased to $67.36 \%$ of its initial value, while the $\mathrm{CL}$ content simultaneously increased to $235.95 \%$ of the initial value at the same point in time. The results provide direct evidence that the glycolidyl and lactidyl units were preferentially lost with degradation for all the PLGC copolymers. Further, the variation in the composition of the PLGC copolymer 


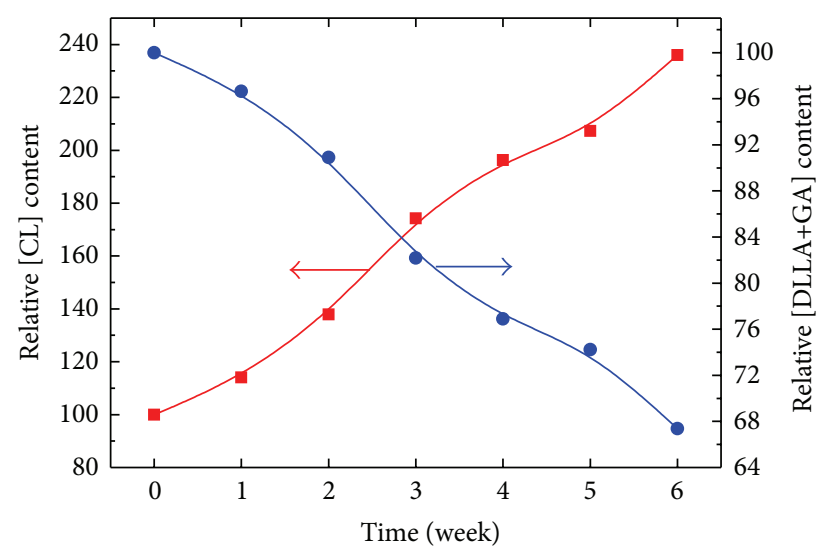

FIGURE 6: The changes in composition of the PLGC copolymer (20 $\mathrm{mol} \% \mathrm{CL})$ in vitro hydrolytic degradation as function of degradation time.

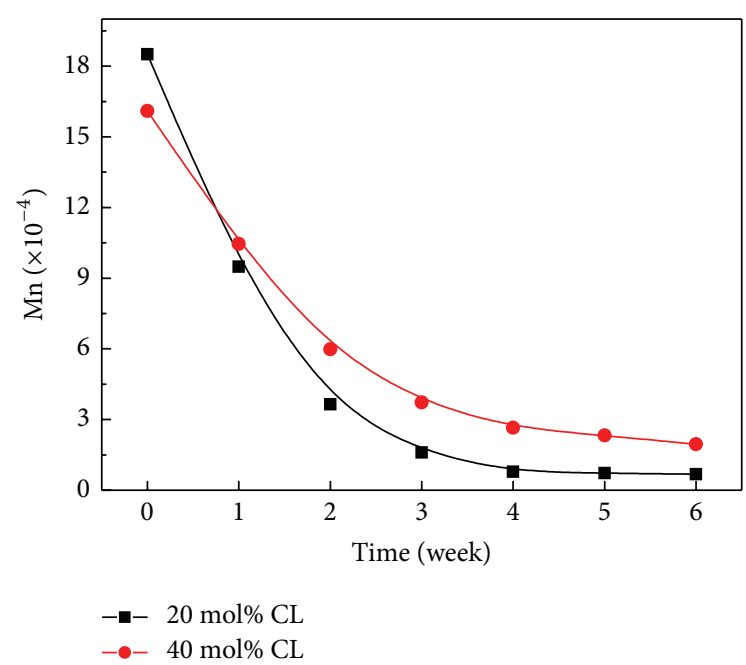

FIGURE 7: The changes in molecular weight of PLGC copolymers in vitro as function of degradation time.

containing $40 \mathrm{~mol} \%$ CL was smaller than that of the PLGC copolymer containing $20 \mathrm{~mol} \% \mathrm{CL}$ because of its slower rate of weight loss.

With the elevated loss in mass and changes in composition, the molecular weights $(\mathrm{Mn})$ of the PLGC copolymers decreased sharply with degradation time (Figure 7). The Mn of the PLGC (20 mol\% CL) samples decreased faster than the copolymers because of greater weight loss. The results showed that PLGC copolymers can be degraded to products with low molecular weights and could eliminate the removal of the materials implanted in vivo when used as a drug matrix. We note that the PLGA 50/50 samples prepared in our study did not dissolve in $\mathrm{CHCl}_{3}$ because of their high molecular weights, and similar observations were found for the PLGA 50/50 samples in primary stages of degradation. Therefore, changes in molecular weights of PLGA 50/50 during hydrolytic degradation were not provided.

We compared the loss of mass with the changes in molecular weight and concluded that the hydrolysis degradation behaviors of PLGC in PBS could be divided into two stages. In the first stage, the molecular weight of PLGC decreased continuously with degradation time, yet little weight loss occurred at this stage. But in the second stage, the molecular weight of PLGC decreased to a low value and remained relatively constant with further degradation while the samples experienced significant weight loss. This was consistent with the common hydrolysis rule of aliphatic polyester that the weight loss would not take place until the molecular weight of samples had decreased to a critical value able to dissolve in water [30].

The morphology change of the PLGC copolymer with degradation was observed by SEM. Figure 8 shows the SEM photographs of the surface of PLGC (20 mol\% CL) before and after in vitro hydrolytic degradation. Before degradation, the surface of the specimens appears rather smooth with some stripes due to the surface morphology of the mold. After 2 weeks, small cracks as well as surface irregularities appeared, indicating the degradation occurred on the surface. As the degradation progressed, the erosion became remarkable and the roughness of the surface increased as it presented a vigorous up-and-down fluctuation profile as shown in Figure 8(c). Figure $8(\mathrm{~d})$ showed the cross section of PLGC $(20 \mathrm{~mol} \%$ CL) after 5 weeks' degradation, which indicates that the degradation occurred not only on the surface, but also inside the polymer. This may have been caused by the autocatalytic effect from the accumulation of acidic degradation product inside the polymer matrix, as was found by previous studies [31-33].

Previous studies determined that acidic degradation products would be produced upon the degradation of PLGA and are detrimental to cells, drugs, or proteins and can lead to inflammatory reactions [14-16]. Many efforts have been devoted to the elimination of the acidic products of the PLGA-based carrier used for osteomyelitis treatment. In this study, the introduction of CL units into the PLGA backbone was demonstrated as an effective strategy to overcome these problems, as shown in Figure 9. The median $\mathrm{pH}$ values decreased as copolymer degradation occurred. This decrease in $\mathrm{pH}$ indicated the continuous release of carboxylic groups into the surrounding media during the hydrolytic degradation process. However, the $\mathrm{pH}$ of the solutions affected by the accumulated degradation products decreased to varying degrees depending on the composition of the PLGC copolymers. For instance, the fastest decrease in $\mathrm{pH}$ was observed for the PLGA with no introduction of CL units, which resulted in a sudden decrease in the $\mathrm{pH}$ of the solutions to 6.46 after 21 days of the in vitro hydrolytic degradation. Faster degradation rates (Figure 5) resulted in more acidic products being released. Compared to PLGA, the PLGC copolymers caused a slower decrease in $\mathrm{pH}$ as the $\mathrm{CL}$ content increased because of the slower degradation rate, especially for the PLGC ( $40 \mathrm{~mol} \% \mathrm{CL})$ that caused a decrease in the $\mathrm{pH}$ of the solutions to 7.11 after 21 days. The results once again confirmed that the CL content in the composition had significant effects on PLGC degradation, and the introduction of CL unit into the PLGA backbone was beneficial to diminish the adverse effects of the degradation product on the clinical applications of PLGA. 

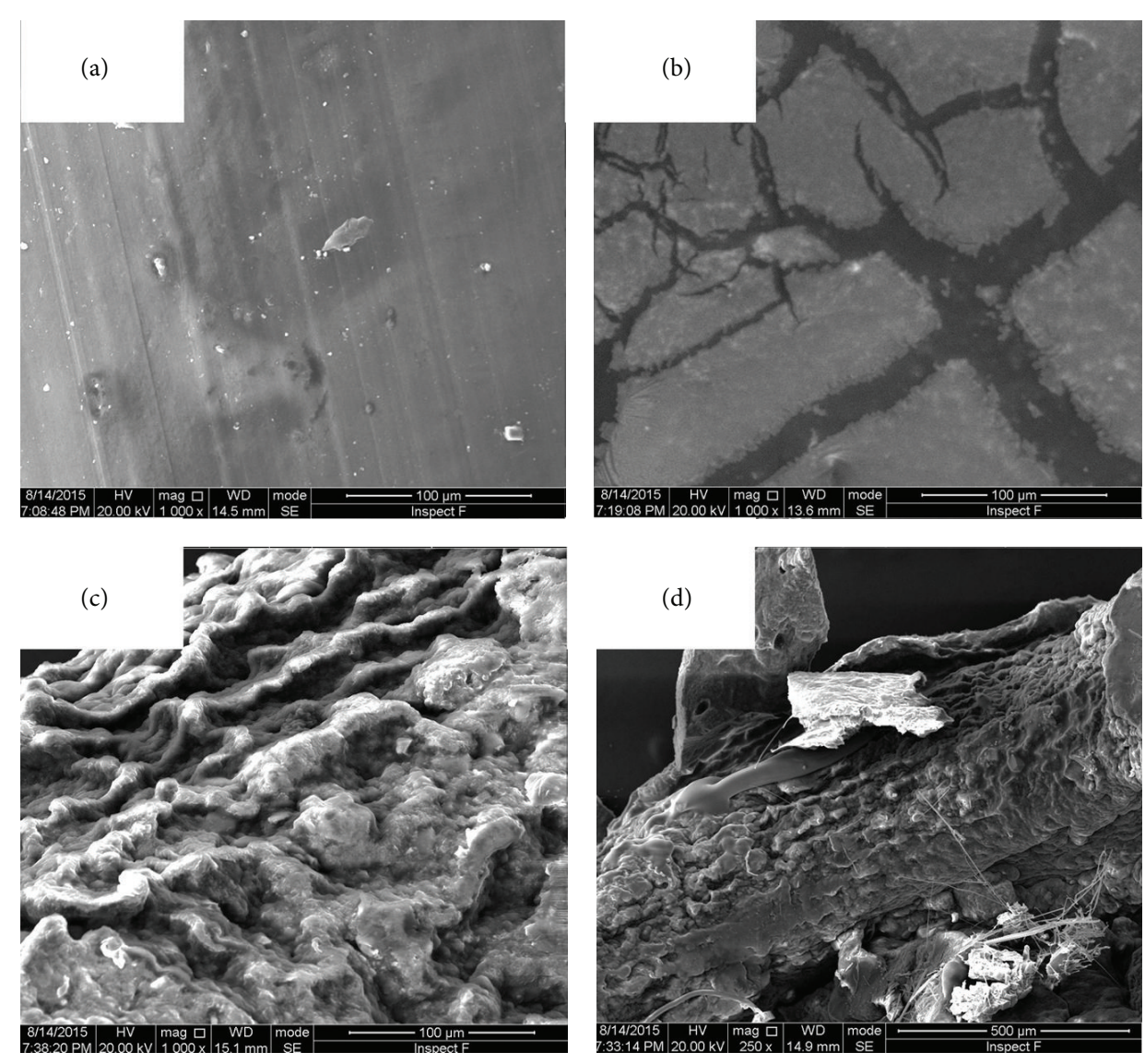

FIGURE 8: The morphology change of PLGC (20 mol\% CL) with in vitro hydrolytic degradation observed under SEM; (a)-(c) surface and (d) cross section; (a) 0 weeks; (b) 2 weeks; (c) and (d) 6 weeks.

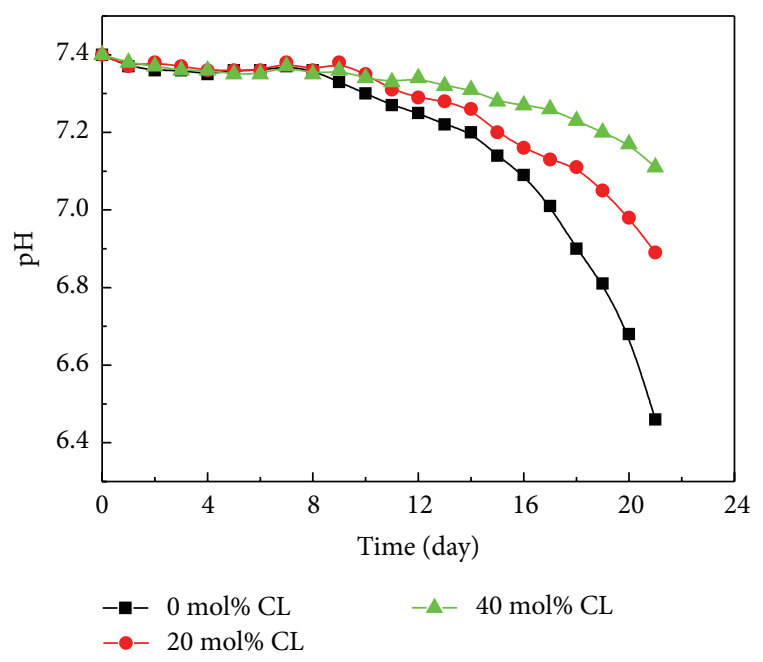

FIGURE 9: The changes in the $\mathrm{pH}$ of PBS solutions during the degradation process of the PLGC copolymers.

\section{Conclusion}

A series of PLGC copolymers with different properties were synthesized by a ring-opening polymerization of DLLA, GA, and CL, using a stannous octoate as catalyst. The CL content had a significant effect on the end properties of PLGC. Depending on the CL content, the properties of the copolymer ranged from stiff materials to elastic polymers, and the thermal properties $\left(T_{g}\right.$ and $T_{d}$ ) and mechanical properties of the copolymer were tailored to meet the requirements for clinical applications. The hydrolytic degradation of the PLGC was performed in $\mathrm{pH}$ 7.4 PBS as compared to PLGA. The degradation rate of the PLGC copolymers was a function of the CL content in composition. Slower degradation rates were detected for copolymers with higher CL contents. The $\mathrm{pH}$ data showed that the PLGC copolymers produced fewer acidic degradation products with increased CL content, which would decrease the inflammation caused by the acidic degradation products released into the surrounding tissues. The in vivo degradation behavior and biocompatibility of the PLGC will be reported later, and further studies will focus on the release behavior and the antibacterial effect of the PLGC implants compounded with Ciprofloxacin.

\section{Competing Interests}

The authors declare that they have no competing interests. 


\section{Acknowledgments}

The authors wish to acknowledge the assistance of Northeastern University for the tests of thermal and mechanical properties.

\section{References}

[1] K. Klemm, "Septopal-a new way of local antibiotic therapy," in Local Antibiotic Treatment in Osteomyelitis and Soft Tissue Infections, T. J. G. Van Rens and F. H. Kayser, Eds., p. 24, Excerpta Medica, Amsterdam, The Netherlands, 1981.

[2] D. H. Robinson, S. Sampath, and M. Pharm, "Release kinetics of tobramycin sulfate from polymethylmethacrylate implants," Drug Development and Industrial Pharmacy, vol. 15, no. 14-16, pp. 2339-2357, 1989.

[3] M. J. Penner, C. P. Duncan, and B. A. Masri, "The in vitro elution characteristics of antibiotic-loaded CMW and Palacos-R bone cements," Journal of Arthroplasty, vol. 14, no. 2, pp. 209-214, 1999.

[4] O. S. Kluin, H. C. Van Der Mei, H. J. Busscher, and D. Neut, "Biodegradable vs non-biodegradable antibiotic delivery devices in the treatment of osteomyelitis," Expert Opinion on Drug Delivery, vol. 10, no. 3, pp. 341-351, 2013.

[5] J. S. McLaren, L. J. White, H. C. Cox et al., "A biodegradable antibiotic-impregnated scaffold to prevent osteomyelitis in a contaminated in vivo bone defect model," European Cells and Materials, vol. 27, pp. 332-349, 2014.

[6] J. Y. Ferguson, M. Dudareva, N. D. Riley, D. Stubbs, B. L. Atkins, and M. A. McNally, "The use of a biodegradable antibioticloaded calcium sulphate carrier containing tobramycin for the treatment of chronic osteomyelitis," Bone and Joint Journal, vol. 96, no. 6, pp. 829-836, 2014.

[7] C. Makarov, V. Cohen, A. Raz-Pasteur, and I. Gotman, "In vitro elution of vancomycin from biodegradable osteoconductive calcium phosphate-polycaprolactone composite beads for treatment of osteomyelitis," European Journal of Pharmaceutical Sciences, vol. 62, pp. 49-56, 2014.

[8] M. Nair and A. Krishnan, "Antibiotic releasing biodegradable scaffolds for osteomyelitis," Current Drug Delivery, vol. 11, no. 6, pp. 687-700, 2014.

[9] V. Yaprakci, O. Erdemli, A. Kayabolen, A. Tezcaner, F. Bozkurt, and D. Keskin, "In vitro/in vivo comparison of cefuroxime release from poly( $\varepsilon$-caprolactone)-calcium sulfate implants for osteomyelitis treatment," Biotechnology and Applied Biochemistry, vol. 60, no. 6, pp. 603-616, 2013.

[10] K. Kimishima, T. Matsuno, J. Makiishi et al., "Effects of gatifloxaine content in gatifloxacine-loaded PLGA and $\beta$-tricalcium phosphate composites on efficacy in treating osteomyelitis," Odontology, vol. 104, no. 1, pp. 105-113, 2016.

[11] V. Uskoković, C. Hoover, M. Vukomanović, D. P. Uskoković, and T. A. Desai, "Osteogenic and antimicrobial nanoparticulate calcium phosphate and poly-(d,l-lactide-co-glycolide) powders for the treatment of osteomyelitis," Materials Science and Engineering C, vol. 33, no. 6, pp. 3362-3373, 2013.

[12] Z. Orhan, E. Cevher, A. Yildiz, R. Ahiskali, D. Sensoy, and L. Mülazimoğlu, "Biodegradable microspherical implants containing teicoplanin for the treatment of methicillin-resistant Staphylococcus aureus osteomyelitis," Archives of Orthopaedic and Trauma Surgery, vol. 130, no. 1, pp. 135-142, 2010.

[13] P. K. Naraharisetti, H. C. G. Lee, Y. C. Fu, D. J. Lee, and C. $\mathrm{H}$. Wang, "In vitro and in vivo release of gentamicin from biodegradable discs," Journal of Biomedical Materials Research Part B: Applied Biomaterials, vol. 77, no. 2, pp. 329-337, 2006.

[14] K. A. Athanasiou, G. G. Niederauer, and C. M. Agrawal, "Sterilization, toxicity, biocompatibility and clinical applications of polylactic acid/polyglycolic acid copolymers," Biomaterials, vol. 17, no. 2, pp. 93-102, 1996.

[15] E. Sachlos and J. T. Czernuszka, "Making tissue engineering scaffolds work. Review: the application of solid freeform fabrication technology to the production of tissue engineering scaffolds," European cells \& materials, vol. 5, p. 29, 2003.

[16] J. M. Karp, M. S. Shoichet, and J. E. Davies, "Bone formation on two-dimensional poly(DL-lactide-co-glycolide) (PLGA) films and three-dimensional PLGA tissue engineering scaffoldsin vitro," Journal of Biomedical Materials Research, vol. 64A, no. 2, pp. 388-396, 2003.

[17] H. Sun, L. Mei, C. Song, X. Cui, and P. Wang, "The in vivo degradation, absorption and excretion of PCL-based implant," Biomaterials, vol. 27, no. 9, pp. 1735-1740, 2006.

[18] H. Seyednejad, D. Gawlitta, R. V. Kuiper et al., "In vivo biocompatibility and biodegradation of 3D-printed porous scaffolds based on a hydroxyl-functionalized poly( $\varepsilon$-caprolactone)," Biomaterials, vol. 33, no. 17, pp. 4309-4318, 2012.

[19] L. Yang, J. Li, Y. Jin, M. Li, and Z. Gu, "In vitro enzymatic degradation of the cross-linked poly( $\varepsilon$-caprolactone) implants," Polymer Degradation and Stability, vol. 112, pp. 10-19, 2015.

[20] M. Ann Woodruff and D. W. Hutmacher, "The return of a forgotten polymer-polycaprolactone in the 21st century," Progress in Polymer Science, vol. 35, no. 10, pp. 1217-1256, 2010.

[21] Y. Habibi, A.-L. Goffin, N. Schiltz, E. Duquesne, P. Dubois, and A. Dufresne, "Bionanocomposites based on poly $(\varepsilon-$ caprolactone)-grafted cellulose nanocrystals by ring-opening polymerization," Journal of Materials Chemistry, vol. 18, no. 41, pp. 5002-5010, 2008.

[22] Y. Baimark, M. Srisa-ard, and A. Puntumchai, "Synthesis of poly (D,L-lactic acid-co-glycolic acid-co-e-caprolactone) terpolyesters by direct polycondensation," Current Research in Chemistry, vol. 2, no. 1, pp. 10-17, 2010.

[23] L. Q. Yang, S. Meng, D. H. Liu et al., "Potential biodegradable implants from $\epsilon$-caprolactone and D, L-lactide copolymers: synthesis, properties, and in vivo degradation," International Journal of Polymer Analysis and Characterization, vol. 19, no. 5, pp. 422-440, 2014.

[24] L. J. Ji, K. L. Lai, B. He et al., "Study on poly(l-lactide-cotrimethylene carbonate): synthesis and cell compatibility of electrospun film," Biomedical Materials, vol. 5, no. 4, Article ID 045009, 2010.

[25] L.-Q. Yang, D. Yang, Y.-M. Guan, J.-X. Li, and M. Li, "Random copolymers based on trimethylene carbonate and $\varepsilon$ caprolactone for implant applications: synthesis and properties," Journal of Applied Polymer Science, vol. 124, no. 5, pp. 3714-3720, 2012.

[26] Y. Shen, Z. Shen, Y. Zhang, and K. Yao, "Novel rare earth catalysts for the living polymerization and block copolymerization of $\varepsilon$-caprolactone," Macromolecules, vol. 29, no. 26, pp. 82898295,1996

[27] T. G. Fox, "Influence of diluent and of copolymer composition on the glass transition temperature of a polymer system," Bulletin of the American Physical Society, vol. 1, no. 3, pp. 123-131, 1956.

[28] D. E. Perrin and J. P. English, "Polyglycolide and polylactide," in Handbook of Biodegradable Polymers, A. J. Domb, J. Kost, and D. 
Wiseman, Eds., p. 3, Harwood Academic Publishers, London, UK, 1998 .

[29] J. Idaszek, A. Bruinink, and W. Święszkowski, "Delayed degradation of poly(lactide-co-glycolide) accelerates hydrolysis of poly( $\varepsilon$-caprolactone) in ternary composite scaffolds," Polymer Degradation and Stability, vol. 124, pp. 119-127, 2016.

[30] T. G. Park, "Degradation of poly(lactic-co-glycolic acid) microspheres: effect of copolymer composition," Biomaterials, vol. 16, no. 15, pp. 1123-1130, 1995.

[31] T. Casalini, F. Rossi, S. Lazzari, G. Perale, and M. Masi, "Mathematical modeling of PLGA microparticles: from polymer degradation to drug release," Molecular Pharmaceutics, vol. 11, no. 11, pp. 4036-4048, 2014.

[32] A. N. F. Versypt, D. W. Pack, and R. D. Braatz, "Mathematical modeling of drug delivery from autocatalytically degradable PLGA microspheres-a review," Journal of Controlled Release, vol. 165, no. 1, pp. 29-37, 2013.

[33] X. Zhu and R. D. Braatz, "A mechanistic model for drug release in PLGA biodegradable stent coatings coupled with polymer degradation and erosion," Journal of Biomedical Materials Research Part A, vol. 103, no. 7, pp. 2269-2279, 2015. 

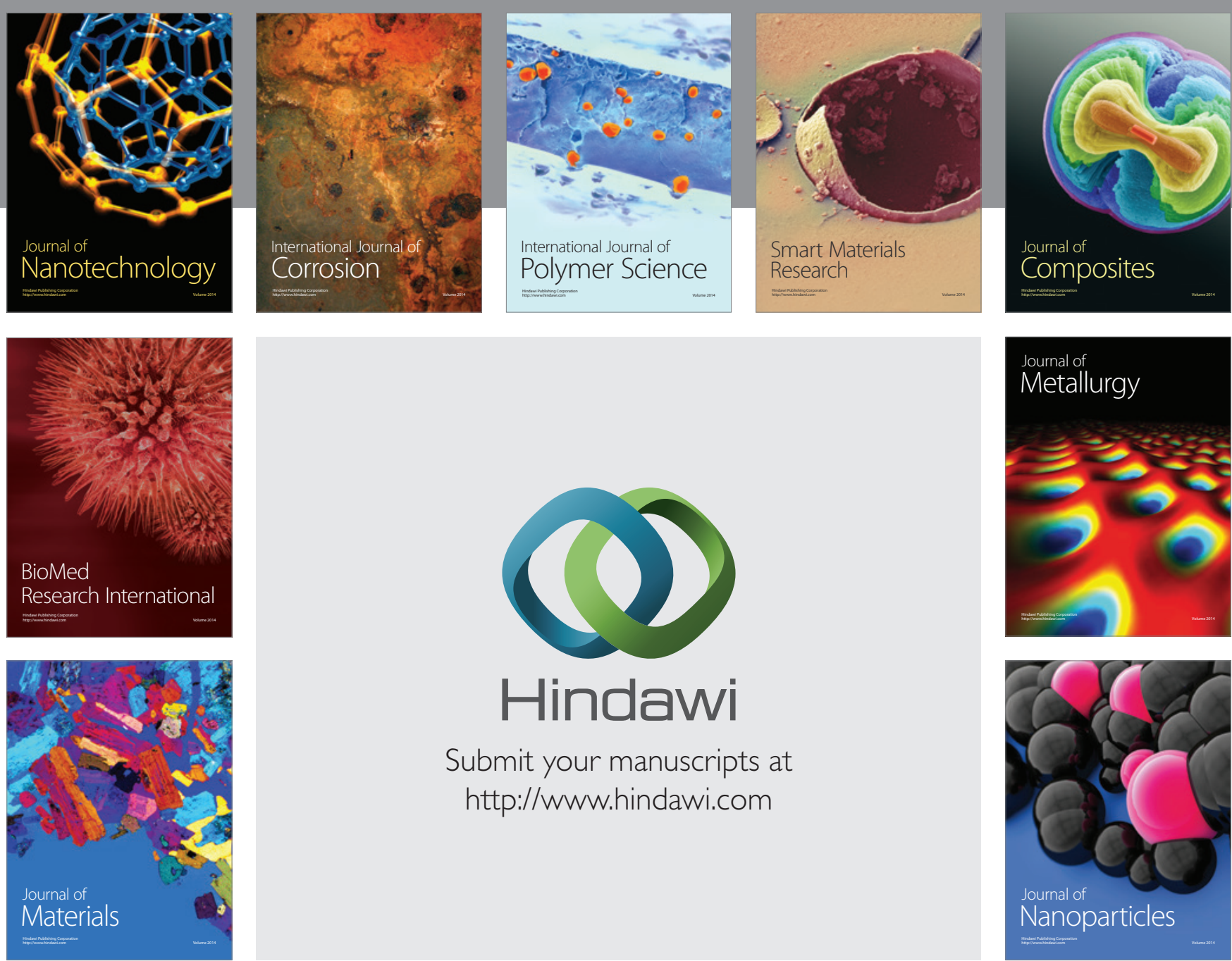

\section{Hindawi}

Submit your manuscripts at

http://www.hindawi.com

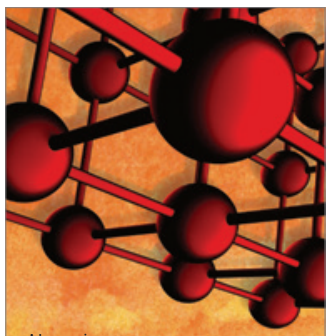

Materials Science and Engineering
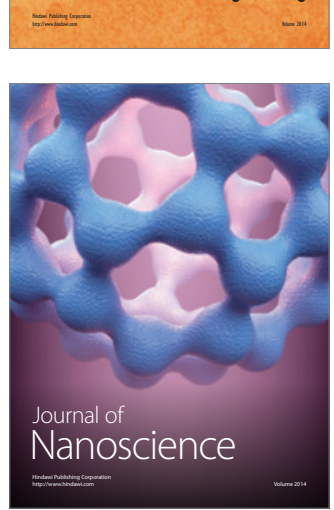
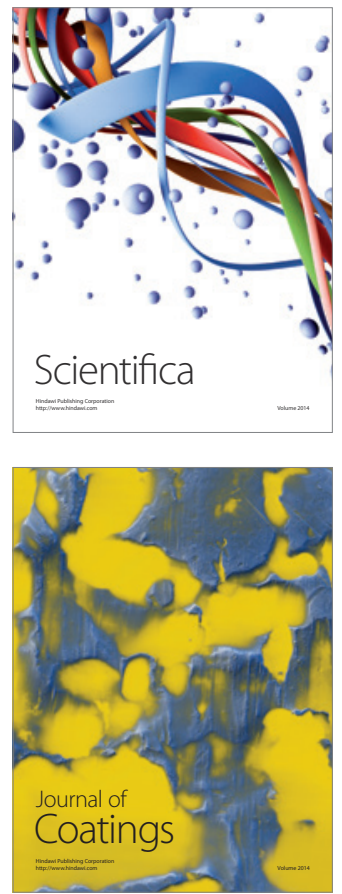
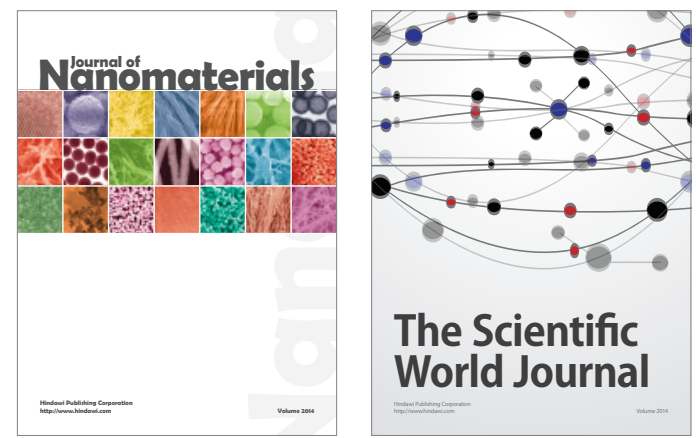

The Scientific World Journal
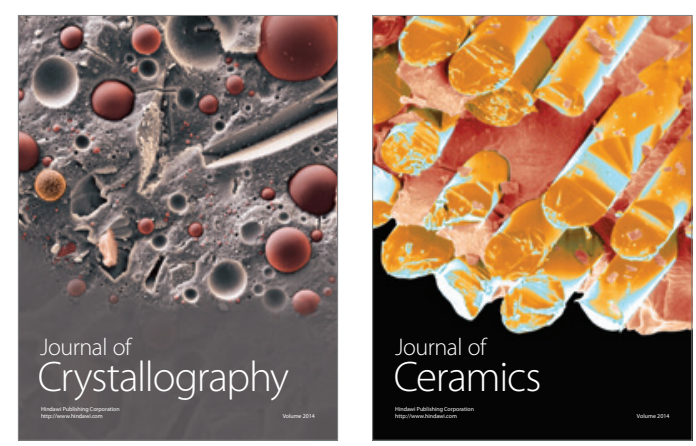
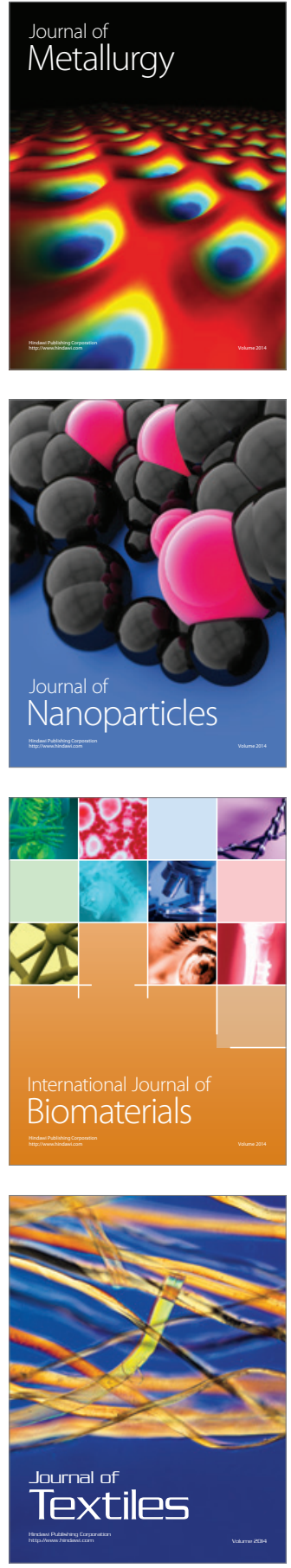